\section{[gw22-e0735] CALPAIN ACTIVATION CONTRIBUTES TO MYOCARDIAL APOPTOSIS INDUCED BY ISCHEMIA-REPERFUSION}

Houxiang Hu Department of Cardiology, Affiliated Hospital of North Sichuan Medical College, Nanchong, China

10.1136/heartjnl-2011-300867.69

Background Calpain is a family of cytoplasmic cysteine proteases activated by calcium ions. The mechanistic link between apoptosis and activation of calpain remains unclear. Apoptotic mechanism have been implicated in myocardial ischemia-reperfusion (I/R) injury.

Aim The aim of this study was to investigate the role of calpain in adult cardiomyocyte apoptosis induced by I/R.

Methods Male wild type mice were randomly divided into control and PD150606 groups. Mice were subjected to myocardial ischemia by occlusion of the left anterior descending coronary artery for $45 \mathrm{~min}$ and reperfusion for $3 \mathrm{~h}$ (I/R). Terminal deoxynucleotidyl transferase d-UTP nick end labelling (TUNEL) staining was performed using an In Situ Cell Death Detection kit on paraffin heart sections $(5 \mathrm{~mm})$. Hoechst 33342 was used as a counter-stain. Adult mouse cardiomyocytes were isolated and cultured, then subjected to ischemia for $1 \mathrm{~h}$, and reperfusion for $3 \mathrm{~h}$. The survival of cardiomyocytes, activity of calpain and caspase-3, cytoplasmic DNA fragments and cytochrome c concentrations were determined.

Results Compared to control, the numbers of TUNEL-positive nuclei were significantly increased in the peri-infarct area in $\mathrm{I} / \mathrm{R}$ mice $(\mathrm{p}<0.05)$. Compared to normal cultured cardiomyocytes, the survival of the cells significantly decreased, however the activation of calpain and caspase-3, and cytoplasmic DNA fragments and cytochrome c concentrations were significantly increased in simulated I/R $(p<0.05)$. These effects of $\mathrm{I} / \mathrm{R}$ on cardiomyocytes were alleviated by calpain inhibitor PD150606 ( $p<0.05)$.

Conclusion Calpain inhibitor PD150606 decreases myocardial apoptosis induced by I/R. Our data suggests that calpain activation may be a critical role in the development of myocardial I/R. 